\title{
Respiratory Motion Modelling and Prediction Using Probability Density Estimation
}

\author{
Majdi R. Alnowam, E. Lewis, M. Guy and K. Wells
}

\begin{abstract}
One of the current major challenges in clinical imaging is modeling and prediction of respiratory motion, for example, in nuclear medicine or external-beam radio therapy. This paper presents preliminary work in developing a method for modeling and predicting the temporal behavior of the anterior surface position during respiration. This is achieved by tracking the anterior surface during respiration and projecting the captured motion sequence data into a lower dimensional space using Principle Component Analysis and extracting the variation in the Abdominal surface and Thoracic surface separately. Modeling is based on learning the multivariate probability distribution of the motion sequence using a joint Probability Distribution Function (PDF) between the variation of the Thoracic surface and Abdomen surface in the Eigen space. Moreover, the prediction model encodes the amplitude of the variation in the Eigen space for both Thoracic surface and Abdominal surface and the derivative of the variation which reflects the motion path (velocity). The joint Probability Distribution Function (PDF) of the prediction model covers the likelihood of each position/phase configuration and the associated maximum-likelihood motion path. Moreover, feeding the real-time tracking data into the model during nuclear medicine acquisition or external-beam radio therapy will facilitate adjusting the model for any changes and overcome irregularities in the observed respiration cycle.
\end{abstract}

\section{INTRODUCTION}

$\mathbf{O}$ $\mathrm{NE}$ of the current challenges in nuclear emission tomography is respiratory motion correction. Respiratory motion during the emission image acquisition process leads to blurred images, thus challenging diagnosis, planning and follow-up processes. There is significant clinical evidence on the effect that respiratory motion has on lesions localization [1], [2], [3], [4]. Correspondingly, significant tumor motion and variation in lung volume has been reported in [5], [6] whereby images reconstructed without respiratory motion compensation were studied. Thus, there exists significant research focus in developing new approaches to monitor and track respiratory motion during data acquisition[7] and understanding the relationship between external marker motion and internal organ motion [8].

This paper presents a new methodology for building a patient specific model for the anterior surface temporal motion during respiration for motion correction application in nuclear medicine and a prediction model for motion correction in external beam

Majdi R. Alnowam, E. Lewis, and K. Wells are from the Centre for Vision, Speech and Signal Processing, FEPS, University of Surrey, Guildford, Surrey GU2 7XH, UK.

M. Guy is from Medway Maritime Hospital, Windmill Road, Gillingham, Kent, ME7 5NY, UK.

Further author information E-mail: m.alnowami@surrey.ac.uk. radiotherapy. This was achieved by tracking the anterior surface temporal motion during respiration using a marker-based tracking system for a set of twenty volunteers and extracting the temporal variation in the Abdominal surface and Thoracic surface separately.

The respiration model is based on learning the multivariate probability distribution of the motion sequence using a joint Probability Distribution Function (PDF) between the variation of the Thoracic surface and Abdominal surface in the Eigen space. Moreover, the prediction model encodes the amplitude of the variation in the Eigen space for both Thoracic surface (TS) and Abdominal surface (AS) and the derivative of the variation which reflects the motion path (velocity). The joint Probability Distribution Function (PDF) of the prediction model covers the likelihood of each position/phase configuration and the associated maximum-likelihood motion path. Moreover, feeding the real-time tracking data into the model during nuclear medicine acquisition or external-beam radio therapy will facilitate adjusting the model for any changes and overcome irregularities encountered in the observed respiration cycle.

\section{Methodology And Results}

\section{A. Volunteer Preparation}

In this study 20 subject participated: 13 male and 7 female. The subjects exhibited significant variation in body shape, as presented by their body mass indices (BMI) ranging between $27.8 \mathrm{~kg} / \mathrm{m}^{2}$ and $21.3 \mathrm{~kg} / \mathrm{m}^{2}$. Subjects then participated in one tracking session for 10 minutes. Each subject was positioned supine on a couch with their arms placed above their head with hands holding contra lateral forearms. This was to simulate the same position during PET acquisition and external beam radiotherapy. They were asked to remain still during the acquisitions and to breathe quietly for 10 $\mathrm{m}$. No others particular instruction regarding the breathing pattern was given.

\section{B. Data Collection}

Each subjects was imaged using a Codamotion infrared markerbased tracking systems. The Codamotion CX1 unit measures the 3D locations of infra-red active markers in real-time. The infrared system Resolution's is about $3 \mathrm{mrad}$ (0.002 degree) at $3 \mathrm{~m}$ from the camera with a lateral resolution of $0.1 \mathrm{~mm}$ and horizontal and vertical resolution of $0.6 \mathrm{~mm}$. The viewing angle of one CX1 unit is approx $80^{\circ}$. The sampling rate of the system was userselected to $10 \mathrm{frame} / \mathrm{sec}$. The markers for this system were arranged on each volunteer's anterior surface in a four-by-four grid ( see Figure 2). These marker arrangement were used to facilitate the ability of modeling and prediction the natural temporal variations in chest wall configuration during breathing, increased accuracy and overcome the problem if irregular breathing pattern. 

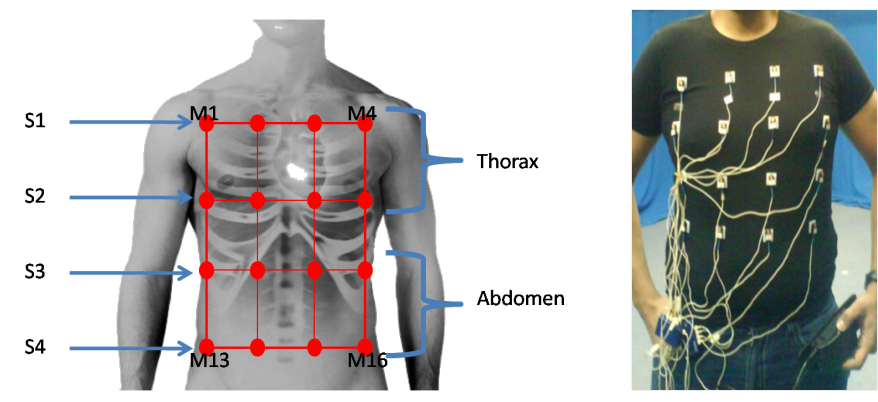

(a) The anterior grid consists of four horizontal (b) Sixteen infrarows and four equally spaced vertical column.

red LED's were placed over the anterior surface of the volunteer

Fig. 1. Tracking points arrangement on each volunteers anterior surface

\section{Motion Capture Results}

In these preliminary analyses, the motion amplitude, (i.e. the displacement between maximum inhalation and maximum exhalation), was used to understand and evaluate the displacement in each marker in the TS and AS marker groups respectively; secondly, the contribution of anterior-posterior (AP) (Z direction), lateral (L) (X direction), and superior-inferior (SI) (Y direction) motion to the total displacement of each marker was analysed. Table ?? summarises the results obtained from scanning the aforementioned volunteer cohort. It shows that the surface displacements for both TS and AS during a breathing cycle where these displacement values describe the average of the total distance each marker travel in the TS or AS. This table demonstrates a mean displacement of the TS (AS) region of $14.5 \pm 7.4 \mathrm{~mm}(22.3 \pm 9.3 \mathrm{~mm})$. In addition, the table indicates that across the volunteer cohort the maximum displacement of TS was $30.6 \mathrm{~mm}$ and the maximum displacement of AS was $39.8 \mathrm{~mm}$.

\section{The Motion Vectors}

For each subjects dataset the capture motion sequences were arranged into two $4 X 2$ groups of markers referred to as the Thoracic surface (TS) and the Abdomen surface (AS) respectively. Each data set (AS and TS) was arranged as a column matrix $F_{n}$ :

$F_{n}=\left[X_{n, 1}, Y_{n, 1}, Z_{n, 1}, X_{n, 2}, Y_{n, 2}, Z_{n, 2} \ldots \ldots \ldots \ldots \ldots, X_{n, s}, Y_{n, s}, Z_{n, s}\right]^{T}$

where $X_{n, 1}, Y_{n, 1}, Z_{n, 1} \ldots . . X_{n, s}, Y_{n, s}, Z_{n, s}$ describe the Cartesian data of each marker and 1 through $s$ is the marker index at frame $n$. As a result each frame will be represented by $D * 1$ vectors, where $D=3 * s$ is the dimensional vectors for $F_{n}$. Therefore, by using 8 markers for the TS and AS respectively, the representation of each data set is a 24 dimensional vector. Figure illustrated the trajectories of the markers displacement of the markers over the acquisition period.

The respiratory motion sequence for each volunteer is represented by a matrix $M=\left[F_{1}, \ldots \ldots \ldots . ., F_{3000}\right]$ for both AS and TS, where each row of the motion matrix corresponds to all the motion components of a particular marker, and each column of $M$ corresponds to a set of measurements from one particular frame.

\section{E. Dimensional Reduction}

Principle component analysis (PCA) was used to reduce dimensionality of the acquired data and analyze the most
TABLE I

MEAN EXPERIMENTAL SURFACE DISPLACEMENTS FOR BOTH CHEST AND ABDOMINAL REGION DURING A BREATHING CYCLE FOR A GROUP OF 10 MALE AND FEMALE VOLUNTEERS.

\begin{tabular}{|c|c|c|c|c|}
\hline $\begin{array}{l}\text { Volunteers } \\
\text { No. }\end{array}$ & Gender & $\begin{array}{l}\text { BMI } \\
\left(\mathrm{Kg} / m^{2}\right)\end{array}$ & $\begin{array}{l}\text { TS Dis- } \\
\text { placement } \\
(\mathrm{mm})\end{array}$ & $\begin{array}{l}\text { AS Dis- } \\
\text { placement } \\
(\mathrm{mm})\end{array}$ \\
\hline 1 & $\mathrm{M}$ & 28.2 & $12.5 \pm 5.1$ & $39.8 \pm 4.9$ \\
\hline 2 & M & 24.3 & $11.3 \pm 2.2$ & $27.9 \pm 3.1$ \\
\hline 3 & M & 25.2 & $24.6 \pm 2.6$ & $8.5 \pm 1.1$ \\
\hline 4 & M & 21.8 & $18.4 \pm 3.1$ & $11.0 \pm 2.3$ \\
\hline 5 & M & 27.8 & $6.8 \pm 1.4$ & $12.7 \pm 2.4$ \\
\hline 6 & M & 24.8 & $10.3 \pm 1.2$ & $10.8 \pm .1 .9$ \\
\hline 7 & M & 21.4 & $13.6 \pm 4.3$ & $25.7 \pm 5.6$ \\
\hline 8 & M & 17.9 & $11.3 \pm 4.3$ & $28.9 \pm 4.5$ \\
\hline 9 & M & 22.2 & $10.1 \pm 1.9$ & $31.1 \pm 3.6$ \\
\hline 10 & M & 24.2 & $21.8 \pm 4.9$ & $31.6 \pm 5.9$ \\
\hline 11 & M & 26.8 & $18.6 \pm 2.5$ & $31.8 \pm 4.1$ \\
\hline 12 & M & 27.2 & $27.0 \pm 4.6$ & $18.0 \pm 2.4$ \\
\hline 13 & M & 25.4 & $30.6 \pm 4.5$ & $35.1 \pm 9.3$ \\
\hline 14 & $\mathrm{~F}$ & 25.2 & $13.5 \pm 1.1$ & $9.6 \pm 2.3$ \\
\hline 15 & $\mathrm{~F}$ & 24.6 & $7.4 \pm 1.2$ & $15.6 \pm 2.3$ \\
\hline 16 & $\mathrm{~F}$ & 22.2 & $7.6 \pm 2.1$ & $22.7 \pm 2.6$ \\
\hline 17 & $\mathrm{~F}$ & 25.5 & $7.1 \pm 2.4$ & $20.3 \pm 2.4$ \\
\hline 18 & $\mathrm{~F}$ & 21.7 & $5.1 \pm 1.4$ & $24.6 \pm 2.8$ \\
\hline 19 & $\mathrm{~F}$ & 23.1 & $10.7 \pm 1.9$ & $25.0 \pm 3.1$ \\
\hline 20 & $\mathrm{~F}$ & 22.5 & $21.3 \pm 2.7$ & $15.7 \pm 1.7$ \\
\hline \multicolumn{2}{|c|}{ Cohort average } & 24.1 & 14.5 & 22.3 \\
\hline \multicolumn{2}{|c|}{ Standard Deviation } & 2.5 & 7.4 & 9.3 \\
\hline \multicolumn{2}{|c|}{$\operatorname{Max}$} & 28.2 & 30.6 & 39.8 \\
\hline \multicolumn{2}{|c|}{ Min } & 17.9 & 5.1 & 8.5 \\
\hline
\end{tabular}

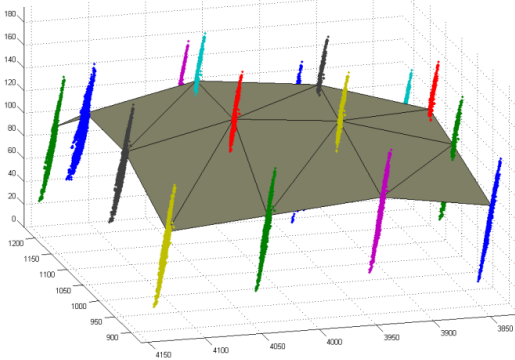

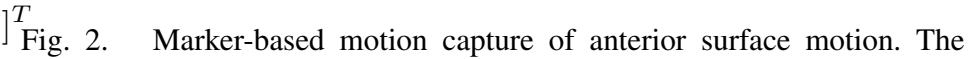
colored trajectories represent the displacement of the markers over the acquisition period. The mesh illustrates the interpolated anterior surface at one particular time instance.

significant aspect of temporal variation without sacrificing significant accuracy. The dimensional reduction was undertaken by extracting the eigenpair (eigenvalues $\lambda_{i}$, eigenvectors $\nu_{i}$ ) of the data covariance matrix $\left.\sigma=\frac{1}{n} \sum_{i=1}^{n}\left(F_{(} i\right)-\bar{F}\right)\left(F_{i}-\bar{F}\right)^{T}$ where $\bar{F}$ is the motion data mean. Once the eigenpairs are determined, these are ranked to gives the variational components in order of highest significance. The number of eigenpairs that are required to describe the original data is dependent on how much of the original variation is described by the first, or first few, eigenvalues. Thus, the dimensionally reduced data for both AS and TS will be represented as a vector $f_{i}$, which re. Figure 3 , shows reflects the varition in the Eigen space for TS and AS. The dominant mode of variation of the AS and TS using the first Eigen-space in 10s 
episodes.

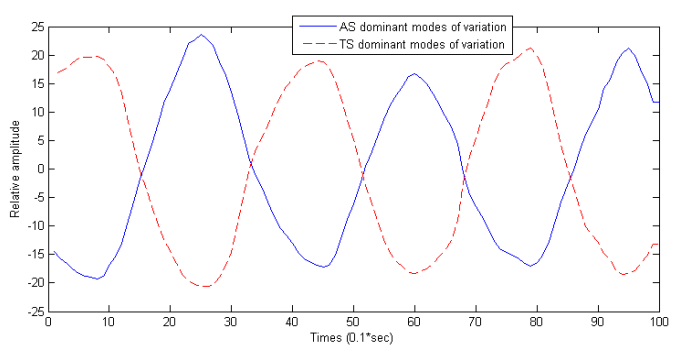

Fig. 3. Illustrates a the dominant modes of variation for the TS and AS in 10s episodes following PCA dimensional reduction where the $\mathrm{x}$-axis represents the number of frame and $y$-axis represent the displacement in $(\mathrm{mm})$

\section{F. The Observation Model}

An observation model of the amplitude of the variation present in the Eigen space can be created using a probability distribution function. In this model a joint Gaussian probability density function between the AS and TS variation in the eigen space are used. These variation can be described by a vector for $T S$ and $A S$ as in equations 2 and 3 .

$$
W_{A S}=\left[W_{A S, 1}, W_{A S, 2}, W_{A S, 3}, W_{A S, 4}, \cdots, W_{A S, n}\right]
$$

$$
W_{T S}=\left[W_{T S, 1}, W_{T S, 2}, W_{T S, 3}, W_{T S, 4}, \cdots, W_{T S, n}\right]
$$

Where $W_{A S}$ and $W_{T S}$ indicate the amplitude of the variation in the eigen space for AS and TS respectively. The joint probability between AS and Ts variation can be described by the following equation.

$$
P\left(W_{T S}, W_{A S}\right)=\frac{1}{n} \sum_{i=0}^{n-1} K_{h}\left(W_{T} S-w_{i}\right) K_{h}\left(\frac{d W}{d t}-\frac{d w_{i}}{d t}\right)
$$

where,$K_{h}(u)$ is abounded kernel function. Illustrated in the following equation:

$$
K_{h}(u)=\frac{1}{h} K\left(\frac{u}{h}\right)=\frac{1}{\sqrt{2 \pi}} e^{\frac{-1}{2}\left(\frac{u}{h}\right)^{2}}
$$

where $h$ is the Gaussian Bandwidth, in this experiment was equal to the standard deviation of the amplitude variation in the eigen space. Figure 4 shows four different breathing model for four different volunteers using a Gaussian kernel Probability Density model (PDE ) which illustrates variety of breathing styles observed in the volunteer data sets. Figure4(a) reflects the relative likelihood of the AS and TS movements: this is described as an in-phase movement pattern. In contrast, Figure 4(b) illustrates an anti-phase movement pattern for different volunteer volunteer. Both of these subjects represent highly consistent respiratory behavior over the 10 minute acquisition period. Figure 4(d) and ?? still reflect the same general pattern of in-phase motion and anti-phase respectively but with much higher degree of variability in the observed pattern.

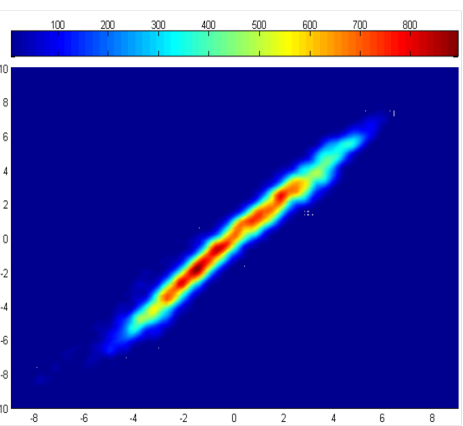

(a) In-phase movement pattern

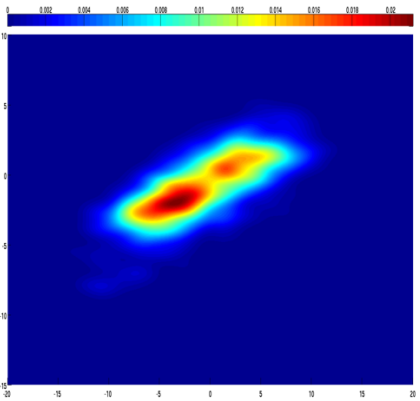

(c) In-consistent in-phase movement

Fig. 4. Illustrates four different breathing model for four different volunteers using a Gaussian kernel Probability Density model (PDE ) which illustrates variety of breathing styles observed in the volunteer data sets. The $\mathrm{X}(\mathrm{Y})$ axis represents the magnitude of the Thoracic(Abdomen) components of the variation vector. reflect that the relative likelihood of the AS (X-axis) vs. TS (Y-axis). The color map illustrates the likelihood of each position with red high and blue low likelihoods.

\section{G. The Prediction Model}

This prediction model will be generated using a Probability Distribution Function (PDF) which encoded both the likelihood of each position/phase configuration and the likelihood of motion from one phase to the next phase. Equation 6 reflects the variation in the Eigen space where Equation 7 reflects the derivative of the variation in the eigen space.

$$
W=\left[w_{1}, w_{2}, w_{3}, w_{4}, \cdots \cdots, w_{n}\right]
$$

$$
\frac{d W}{d t}=\left[\frac{d w_{1}}{d t}, \frac{d w_{2}}{d t}, \frac{d w_{3}}{d t}, \cdots, \frac{d w_{n-1}}{d t}\right]
$$

Real-time tracking during applications such as intensity modulated radiotherapy or imaging patient with nervous ticks or tremor, will facilitate a method of readjusting the model prediction for any changes in the respiration pattern and overcome sharp changes in respiration cycle.

The second PDF encodes the likelihood of the motion form one phase to the next in the reduced eigenspace as in Equation 8. Therefore, this model will not only relay in the likelihood of the position only but also consider the velocity/time information to model the respiratory cycle. The Joint density will be calculated similar to the Equation 4.

$$
P\left(W, \frac{d W}{d t}\right), \text { where } \frac{d W}{d t}=W_{i+1}-W i
$$




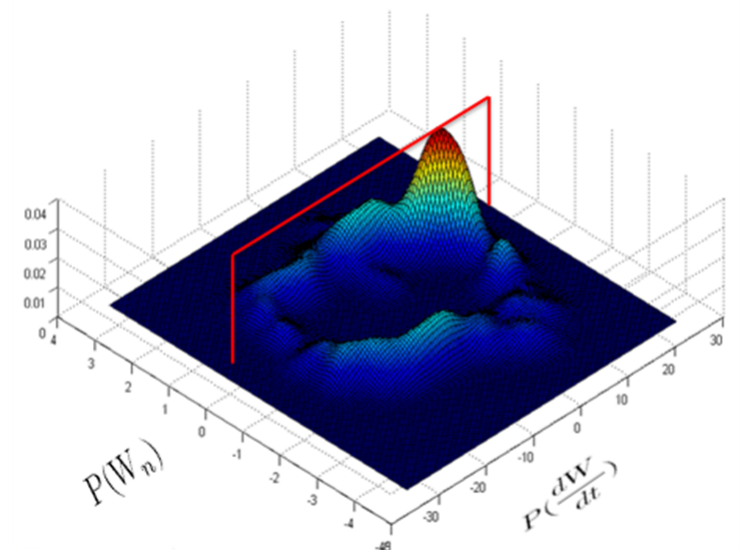

Fig. 5. Illustrates a 3D plot for the Joint PDF between the amplitude of the variation in the Eigen space and the derivative of the variation. The red box illustrate the latest known position $W n$.

Subsequently, by learning the PDF of both factors motion and velocity for all dimensionally reduced motion sequence and taking the latest known potion before prediction as the prediction point.

The highest likelihood movement at this particular position will be found using the combined PDF as illustrated in equation 9:

$$
\frac{\widehat{d W}}{d t}=\arg _{\forall\left(\frac{d w}{d t}\right)} \max \left(P\left(w_{n}, \frac{d W}{d t}\right)\right)
$$

The next position of the frame during the respiration cycle $W_{n+1}$ then constructed using the equation

$$
\widehat{W_{n+1}}=w_{n}+\frac{\widehat{d W}}{d t}
$$

\section{H. The Sliding Window}

To simplify the prediction model, increase the accuracy, and overcome the affect of mean drift or any sharp change in a particular frame, we used a sliding window for adaptive on-the-fly training. Thus, only a 10 second sliding window episode or motion capture sequence immediately preceding the prediction point was used in the prediction model. This sliding window thus rebuilds the prediction model as new capture data become available. Figure 6 illustrates the temporal change in the model as the sliding window evolves with time.
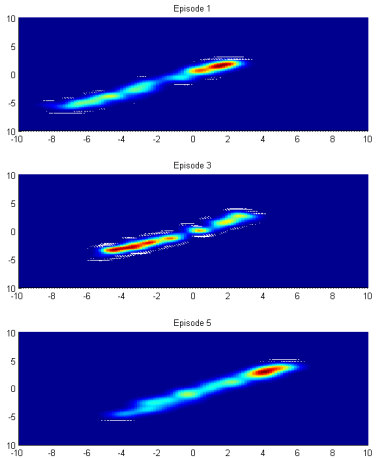
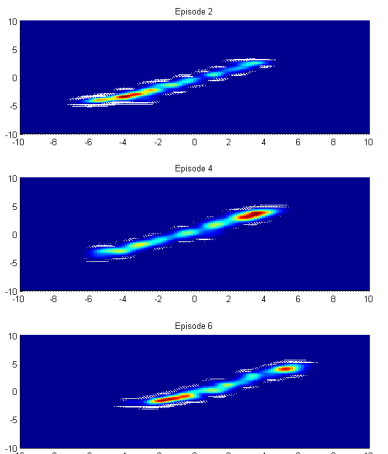

Fig. 6. Illustrate the relative likelihood of the AS (X-axis) vs. TS (Y-axis) movements, in 6 episode for subject 'A4' .

Figure 7 illustrates an example of the prediction model, where $W_{T S}$ and $W_{A S}$ indicate the amplitude of the variation in the Eigen space for TS and AS respectively. After using the first 10 second episode of the data for initial learning, the model start to predict future positions with a look a head period of $0.2 \mathrm{~s}$.

Figure 8 Illustrates the error associated with the prediction model example in Figure 7. The error in the predicted amplitude of the $W_{T S}$ and $W_{A S}$ variation in the Eigen space are indicated by red and blue line respectively. The average Root mean square error associated with this prediction model is $0.78 \mathrm{~mm}$.

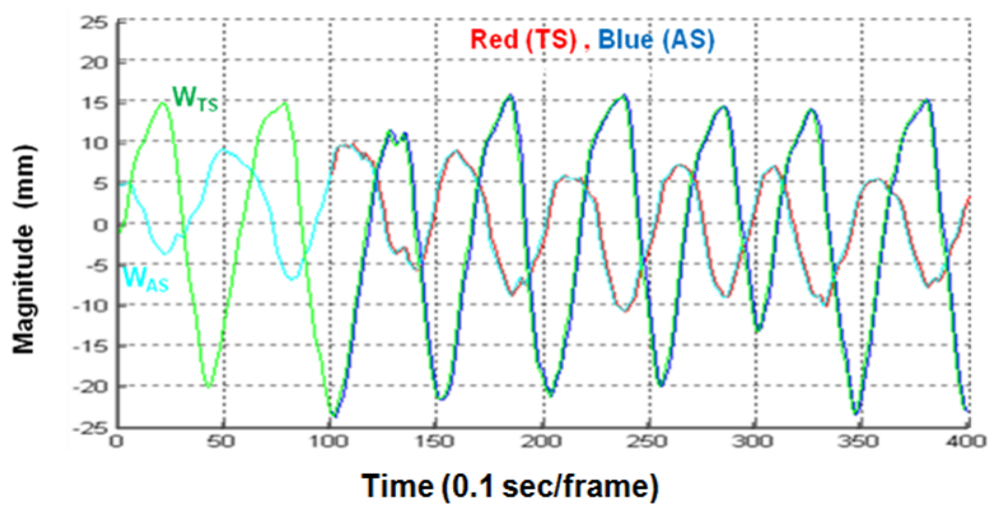

Fig. 7. Illustrates an example of the prediction model. The $W_{T S}$ and $W_{A S}$ indicate the amplitude of the variation in the Eigen space. After learning the first $10 \mathrm{sec}$ the system start the prediction for the future position.

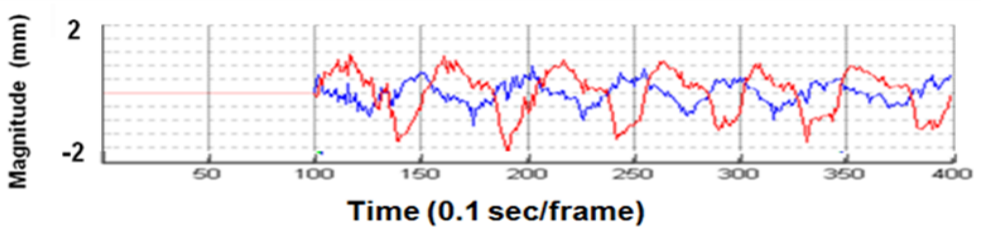

Fig. 8. Illustrates the error associated with the prediction model example in Figure 7. The error in the predicted amplitude of the $W_{T S}$ and $W_{A S}$ variation in the Eigen space are indicated by red and blue line respectively indicate. After learning the first $10 \mathrm{sec}$ the system start prediction for the future position.

\section{DisCUSSION AND CONCLUSION}

These initial results show a promising approach to model the respiratory motion using PDF encoding of both the likelihood of each position/phase configuration and the likelihood of motion from one phase to the next. However, unacceptable levels of prediction error are produced if the estimation based on large parts or the entire global dataset for a particular individual as the affect of sharp changes will be destroyed by the kernel estimation. Therefore, a 10 second sliding window has been used to generate a prediction model with on-the-fly training. The average Root Mean Square error associated with the prediction model was about $0.78 \mathrm{~mm}$. Further development of this work involves building a correlation model between the anterior surface motion and internal organ motion for diagnostic and treatment applications.

\section{REFERENCES}

[1] M. V. Knopp and H. G. Bischoff Radiologe 34(10), pp. 588-91, 1994.

[2] S. A. Nehmeh, Y. E. Erdi, C. C. Ling, K. E. Rosenzweig, H. Schoder, S. M. Larson, H. A. Macapinlac, O. D. Squire, and J. L. Humm J Nucl Med 43(7), pp. 876-81, 2002. 
[3] S. A. Nehmeh, Y. E. Erdi, K. E. Rosenzweig, H. Schoder, S. M. Larson, O. D. Squire, and J. L. Humm J Nucl Med 44(10), pp. 1644-8, 2003.

[4] J. Daouk, L. Fin, P. Bailly, and M. E. Meyer Acta Radiol 50(2), pp. $144-55,2009$.

[5] S. A. Nehmeh, Y. E. Erdi, T. Pan, E. Yorke, G. S. Mageras, K. E. Rosenzweig, H. Schoder, H. Mostafavi, O. Squire, A. Pevsner, S. M. Larson, and J. L. Humm Med Phys 31(6), pp. 1333-8, 2004.

[6] M. Reyes, G. Malandain, P. M. Koulibaly, M. A. Gonzalez-Ballester, and J. Darcourt, "Model-based respiratory motion compensation for emission tomography image reconstruction," Physics in Medicine and Biology 52(12), pp. 3579-3600, 2007.

[7] A. Rahmim, O. Rousset, and H. Zaidi 2, pp. 251-266, Apr. 2007.

[8] R. Boutchko, B. W. Reutter, D. Saloner, and G. T. Gullberg, "Correlating motion of internal organs with the displacements of fiducial markers during respiration," in Nuclear Science Symposium Conference Record, 2008. NSS '08. IEEE, pp. 3641-3642, Oct. 2008. 